\title{
Role of fibreoptic bronchoscopy and CT guided FNAC in diagnostic evaluation of Non resolving Pneumonia with special emphasis on clinical outcome
}

\author{
Bhupendra Kumar Jain', Pooja Sharma², Ashok Bajpai ${ }^{3}$, Satish Motiwale ${ }^{4}$, Nikhilesh Pasari5 ${ }^{5}$, Deepika Patel ${ }^{6}$ \\ ${ }^{1}$ Assistant Professor, ${ }^{2}$ Senior Resident, and ${ }^{3,4}$ Professor, ${ }^{5,6}$ Resident, Department of Respiratory Medicine, Sri Aurobindo Medical College and \\ PG Institute, Indore-Ujjain State Highway, Near MR10 Crossing Indore, Madhya Pradesh - 452004, India
}

\section{A B S TR A C T}

\begin{abstract}
Background: Nonresolving pneumonia is a pneumonia with a delayed or slow resolution of radiographic infiltrates or clinical symptoms despite adequate treatment with antibiotics for a minimum period of 10 days. Fibreoptic bronchoscopy (FOB) and CT guided FNAC has a specific role in diagnosis of Non resolving pneumonia. Aims and Objectives: To assess the diagnostic efficacy of fibreoptic bronchoscopy (FOB) and computed tomography (CT)guided fine needle aspiration cytology (FNAC) in evaluation of non-resolving or slowly resolving pneumonia with special emphasis on clinical outcome. Material and Methods: The study was designed as a prospective observational study. We reviewed FOB in Sixty-five consecutive patients of Non-resolving pneumonia admitted under Respiratory Medicine unit of Sri aurobindo medical college and PG Institute Indore from June 2012 to May 2014. We also reviewed role of CT guided FNAC in selected case where FOB result was inconclusive. Result: Out of total Sixty-five case of non resolving pneumonia, Fifty-two patient (81\%) were diagnosed with the help of FOB and Eleven patients $(91 \%)$ were diagnosed with help of CT guided FNAC out of Twelve patient subjected for procedure. Conclusion: FOB should be the first option before CT-guided FNAC in evaluating non-resolving pneumonia. Both the procedures were safe and no major complication was observed.
\end{abstract}

Key words: Non resolving pneumonia, Fibreoptic Bronchoscopy (FOB), CT guided Fine needle aspiration cytology (CT guided FNAC), Transbronchial lung biopsy (TBLB)

\section{INTRODUCTION}

Kirtland and Winterbauer defined slowly resolving community acquired pneumonia in immunocompetent patients among patients who had improved clinically and defervesced with antibiotic therapy less than $50 \%$ clearing by 2 weeks or less than complete clearing at 4 weeks. ${ }^{1}$ Fein et al defined nonresolving pneumonia as a clinical syndrome in which "focal infiltrates clearly begin with some clinical association of acute pulmonary infection (that is, fever, expectoration, malaise and/or dyspnea) and do not resolve in the expected time". ${ }^{2}$ The expected time of radiographic resolution is influenced by both host factors and the culprit pathogen. In a later review,
Fein and Feinsilver modified their definition to require "a minimum of 10 days of antibiotic therapy and a radiographic infiltrate that has not resolved in an 'expected' period of time based on the presumed diagnosis". ${ }^{3}$ Causes of non resolution pneumonia are co-morbid illnesses like diabetes, alcoholism, COPD and bronchiectasis, age greater than 50 year, cytotoxic/immunosuppressive therapy, bacteremia, multilobar pneumonia, presence of drug resistant organisms, presence of unusual organisms and diseases mimicking pneumonia. Approximately 90 percent of patients younger than 50 years of age show radiographic resolution by four weeks, compared with only 30 percent of patients older than 50 even in the absence of concurrent disease. ${ }^{4}$ The aim of present study was to 
assess the diagnostic efficacy of Fibreoptic bronchoscopy (FOB) and computed tomography (CT)-guided fine needle aspiration cytology (FNAC) in evaluation of non-resolving or slowly resolving pneumonia with special emphasis on clinical outcome.

\section{MATERIAL AND METHODS}

The study was designed as a prospective observational study. We reviewed FOB in Sixty-five consecutive patients of Non-resolving pneumonia admitted under Respiratory Medicine unit of Sriaurobindo Medical College and PG Institute Indore from June 2012 to May 2014. We also reviewed role of CT guided FNAC in selected case where FOB result was negative. Patients admitted with pneumonia not showing adequate clinical and chest radiograph improvement after 10 days of antibiotics were included in the study. A total of 510 patients were admitted with provisional diagnosis of pneumonia, out of which sixtyfive patients, Forty-eight males (73.8\%) and seventeen females $(26.1 \%$ ), who showed less than $50 \%$ of radiological clearance were selected.

Inclusion criteria: Persistence of clinical symptoms and signs cough, fever more than $100^{\circ} \mathrm{F}$, dyspnoea or chest pain) failure of resolution of the radiological focal infiltrate by $50 \%$ in 2 weeks at 2 consecutive chest radiograph despite the antibiotic therapy as per IDSA guidelines for a minimum period of 10 days. Detailed history and physical examination was performed and comorbidities were noted.

Exclusion criteria: Patient who are sputum-positive pulmonary tuberculosis or known case of lung cancer, recent history of myocardial infarction, positive test result for human immunodeficiency virus (HIV) infection, post spleenectomy patient and unwilling patients were excluded from our study.

Blood for complete hemogram, Blood culture, Renal function test, Liver function test, Prothrombin time with INR \& APTT, Chest X-rays (posteroanterior and lateral view), sputum for AFB, sputum for malignant cells and stool for ova \& cyst were sent in all patients. Blood for RA factor, ANA, cANCA and pANCA were done additionally in selected patients wherever found relevant. Macroscopic appearance of bronchial tree during FOB (intraluminal growth or presence of secretions) was noted. Bronchoalveolar lavage (BAL) fluid was sent for cell type, AFB smear and culture for Mycobacterium Tuberculosis (MGIT-960), gram stain and culture, fungal stain and culture and cytology for malignant cells in all patients. In selected case BAL for legionella culture was also done on special media, buffered charcoal yeast extract
(BCYE) agar supplemented with $\alpha$-ketoglutarate. Bronchial brushing and biopsy were also done in relevant patients and were sent for AFB smear, cytology for Malignant cell and histopathology respectively. Patient with no intraluminal growth or inflammation or purulent secretion on FOB were subjected for Transbronchial lung biopsy (TBLB). Post-bronchoscopic sputum was sent for AFB smear and malignant cell. Patients having atypical chest radiograph and who did not give consent for FOB or where results of FOB were inconclusive, were further evaluated by contrast enhanced CT Thorax and CT-guided FNAC was done in relevant cases and FNAC tissue was sent for Histopathology.

\section{SETTING AND ANALYSIS}

Data were analyzed using computer software, Statistical Package for Social Sciences (SPSS) version 10. Data is expressed in its frequency and percentage. To verify the associations and comparisons between different parameters, Fisher Exact test was used as nonparametric test. For statistical evaluations a $\mathrm{p}$ value $<0.05$ was considered significant.

\section{RESULT}

Sixty Five patients were enrolled in the study. Mean age was 51 year with Male: Female ratio of 2.8:1 with Forty-eight males $(73.8 \%)$ and seventeen females $(26.1 \%)$ females (Table 1).

The most common symptoms and sign are cough sixtytwo patients (95\%), Fever fifty-five patients $(84.6 \%)$, Hemoptysis thirty patients (46\%), Chest pain twenty-eight patients (43\%), shortness of breath twenty patients (30.7\%), clubbing eighteen patients $(27.6 \%)$ and Lymphadenopathy four patients $(6.1 \%)$.

Right upper lobe $(35 \%)$ was most commonly involved followed by right lower lobe (21\%) and left upper lobe $(18 \%)$ on chest radiograph (Table 1). Consolidation was present in thirty-nine patients $(60 \%)$ on chest radiograph, consolidation with bronchiectasis was present in twelve patients $(18.4 \%)$, consolidation along with cavity was present in seven patients $(10.7 \%)$, consolidation with airfluid level in cavity was present in four patients $(6.15 \%)$ and only cavity were present in three patients $(4.6 \%)$.

Presence of cavitary lesion was commonest with tuberculous etiology $(n=8)$ but also noted two patients of squamous cell cancer. One case of wegners granulomatosis was also noted which was cANCA positive and chest radiograph revealed multiple cavities with Air-fluid level. Rest four cases of Bronchiectasis were identified by CT Thorax. 
Most common cause of nonresolution of pneumonia in this study was pyogenic infection Twenty-four patient (37\%) followed by tuberculosis Nineteen patients $(29.2 \%)$ and malignancy Fifteen (23\%) (Table 2).

Bronchoalveolar lavage (BAL) culture and sensitivity was helpful in the diagnosis of Pneumococccus Six patients (25\%), Pseudomonas Four patients (16.6\%), Klebsilla Four patients (16.6\%), Legionella Four patient (16.6\%), Acinetobacter Two patients (8.3\%), Staphylococcus Two patients $(8.3 \%)$ and Candida One patient $(4.1 \%)$. BAL for Legionella culture was positive in Four patient out of total Twelve patient subjected for culture. Candida was diagnosed by TBLB Specimen on Fungal culture in one patient and other case was diagnosed by BAL fungal culture.

Tuberculosis was dignosed with BAL AFB Staining in Eight patient, BAL AFB Culture in Five patient, FOB transbronchial biopsy in Two patient and Four patient were diagnosed by CT guided FNAC on histopathology.

Tubercular pneumonia was diagnosed in Twelve patient of diabetes mellitus and rest Seven case of diabetes mellitus were isolated as bacterial pneumonia and One case of diabetes was present in malignant group.

Total Fifteen (23\%) case of Bronchgenic carcinoma were diagnosed (Table 3). Four patients were diagnosed with the help of BAL Cytology, two patients with endobronchial biopsy and two cases with transbronchial

\begin{tabular}{lcc}
$\begin{array}{l}\text { Table 1: Chest } \mathbf{X} \text { ray pattern involvement: Non } \\
\text { resolving pneumonia }\end{array}$ \\
\hline $\mathbf{X}$ ray pattern & $\mathbf{N}=\mathbf{6 5}$ & $\%$ \\
\hline Right upper lobe & 23 & $35 \%$ \\
Right lower lobe & 14 & $21 \%$ \\
Left upper Lobe & 12 & $18 \%$ \\
Multilobar & 8 & 12.3 \\
Left lower Lobe & 6 & $9.2 \%$ \\
Right middle lobe & 2 & $3 \%$ \\
\hline
\end{tabular}

\begin{tabular}{|c|c|c|}
\hline Etiology diagnosis & No. of patient & Patient \% \\
\hline Pyogenic infection & 24 & $37 \%$ \\
\hline Pulmonary tuberculosis & 19 & $29.2 \%$ \\
\hline Bronchogenic carcinoma & 15 & $23 \%$ \\
\hline Foreign body & 2 & $3 \%$ \\
\hline Segmental bronchial stenosis & 1 & $3 \%$ \\
\hline Multiple blood clots & 1 & $1.5 \%$ \\
\hline Wegners granulomatosis & 1 & $1.5 \%$ \\
\hline Total diagnosed & 63 & $96.7 \%$ \\
\hline Undiagnosed & 2 & $3 \%$ \\
\hline
\end{tabular}

lung biopsy while rest seven cases were diagnosed with the help of CT guided FNAC.

Squamous cell Carcinoma were diagnosed in eight patients followed by Adenocarcinoma in four patients, small cell carcinoma in two patients and one patient with large cell carcinoma. Squamous cell carcinoma was diagnosis by endobronchial biopsy in three patients, CT guided FNAC three patients and BAL cytology was helpful in two patients. Adenocarcinoma was diagnosed in four patients. Diagnosis was done by CT guided FNAC in three patients and TBLB was helpful in one patient. Small cell carcinoma was diagnosed by TBLB in one patient and BAL cytology was helpful in diagnosis of one patient. Large cell carcinoma was diagnosed in one patient with help of CT guided FNAC. CT guided FNAC was inconclusive in one patient but patient responded to empirical starting antituberculous drug and infiltrate start regressing in six weeks.

Comorbidities like Smoking, Diabetes Mellitus, Bronchiectasis, Alcoholism were significantly associated with non resolving pneumonia ( $\mathrm{p}$ value $<0.5$ ) in our study while hypertension was not associated with Non resolving pneumonia (Table 4).

In our series mean age involvement was 51 year ( $>50$ year) and smoking was seen in twenty-five patients (38.4\%). Smoking was more significantly associated with malignancy then infectious etiology group ( $p$ value 0.039). Diabetes was found in Twenty patient $(30.7 \%)$ and was more significantly associated with infectious etiology group than malignancy ( $\mathrm{p}$ value 0.01 ). Bronchiectasis was found in sixteen $(26.1 \%)$ of the patient and was more significantly associated with infectious etiology group than malignancy ( $p$ value 0.044 ). Alcoholism was significantly associated with infectious etiology group than malignant group ( $\mathrm{p}$ value 0.044). Hypertension was not associated with malignancy or etiology group ( $\mathrm{p}$ value 0.48 ).

FOB was helpful in diagnosis among fifty-two patients $(81.25 \%)$ out of total sixty-four patient and negative consent for FOB was given by one patient. Computerized tomography guided FNAC was helpful in diagnosis of eleven patients $(91.6 \%)$ out of total twelve patients of non resolving pneumonia subjected for procedure and negative consent for CT guided FNAC was given by one patient who was started on empirical anti-tubercular drug and responded to drugs.

We were able to diagnose sixty-three cases $(96.9 \%)$ with help of FOB and CT guided FNAC. Despite FOB and CT guided FNAC no diagnosis was made in two cases. 


\begin{tabular}{lccccc} 
Table 3: Bronchogenic carcinoma diagnosis & \\
$\begin{array}{l}\text { Bronchogenic } \\
\text { carcinoma }\end{array}$ & $\mathbf{N = 1 5}$ & $\begin{array}{c}\text { FOB bal } \\
\text { cyto-logy }\end{array}$ & $\begin{array}{c}\text { FOB endo-bronchial } \\
\text { biopsy }\end{array}$ & $\begin{array}{c}\text { FOB trans-bronchial } \\
\text { lung biopsy }\end{array}$ & $\begin{array}{c}\text { CT guided } \\
\text { FNAC }\end{array}$ \\
\hline Squamous cell & 8 & 3 & 2 & 0 & 3 \\
Adenocarcinoma & 4 & 0 & 0 & 1 & 3 \\
Small cell & 2 & 1 & 0 & 0 & 0 \\
Large cell & 1 & 0 & 2 & 2 & 7 \\
Total & 15 & 4 & 2 & & 0 \\
\hline
\end{tabular}

\begin{tabular}{|c|c|c|c|c|}
\hline Comorbidities & $\mathbf{N}$ & $\%$ & $\begin{array}{l}\text { Fisher exact } \\
\text { test } p \text { value }\end{array}$ & $p$ value $<0.05$ \\
\hline Smoking & 25 & $(38.4 \%)$ & 0.039 & Significant \\
\hline Diabetes mellitus & 20 & $(30.7 \%)$ & 0.01 & Signifiant \\
\hline Bronchiectasis & 16 & $(24.6 \%)$ & 0.045 & Significant \\
\hline Alcoholism & 17 & $(26.1 \%)$ & 0.044 & Significant \\
\hline Hypertension & $! 2$ & $(18.4)$ & 0.48 & Not significant \\
\hline
\end{tabular}

\section{DISCUSSION}

It accounts for $10 \%$ - $15 \%$ of nosocomial pneumonias and is estimated to be responsible for approximately $15 \%$ of inpatient pulmonary consultation and $8 \%$ of bronchoscopies. ${ }^{5}$ Inadequate knowledge regarding the expected clinical course and outcome of a communityacquired or nosocomial pneumonia is a common reason for pulmonary consultation, selection of patients and appropriate timing of further evaluation can be challenging. ${ }^{6}$

Several studies in the past reported that failure to resolve a community-acquired pneumonia occurs in from 13 to 26 percent of patients. ${ }^{7,8}$ In our series a total of 510 patients were admitted with provisional diagnosis of pneumonia, out of which Sixty-five patients (12.7\%) were isolated as non resolving pneumonia. Most pneumonias will resolve radiologically over two to three months after rational treatment.

Legionnaires disease and bacteraemic pneumococcal pneumonia cleared more slowly than other pneumonias. ${ }^{9}$ In our series also the most common cause of pyogenic non resolving pneumonia was pneomococcus, in six patients $(25 \%)$ followed by legionalla pneumonia in four patients, followed by klebsiella in four patients and pseudomonas in four patients.

Earlier study reported the common conditions associated with delayed resolution are advanced age, COPD, and alcoholism. ${ }^{10}$

Souweine and colleague confirmed that bronchoscopy can identify the bacteria responsible for no responsiveness to therapy in patients with ventilator associated pneumonia. ${ }^{11}$
When endobronchial anatomy is normal and there is no purulence to suggest infection TBBs should be done to exclude noninfectious causes or infections attributable to mycobacteria or fungi. ${ }^{12}$ In our series also TBLB was helpful in diagnosis of two case of tubercular pneumonia, one case of candida pneumonia and two cases of bronchogenic carcinoma.

"Objective data suggest that fiberoptic bronchoscopy with bronchoalveolar lavage and transbronchial biopsy can successfully diagnose approximately $90 \%$ of patients who eventually have a specific diagnosis established. It is most likely to be useful in younger, nonsmoking patients with multilobar involvement and prolonged disease, whereas elderly patients, smokers, and those with immunodeficiency are more likely to have a non diagnostic bronchoscopy and to have a slowly resolving pneumonia. ${ }^{13}$ In this study the diagnostic yield of FOB was $(81.25 \%)$ which is slightly less than other studies. It can be explained on basis that FOB is more helpful in diagnosis of centrally situated non resolving pneumonia and for peripheral situated lesion like adenocarcinoma and large cell bronchogenic carcinoma, CT guided FNAC is best option.

Figure 1 is showing Chest rontgenograph of patient having consolidation left lower lobe and Figure 2 is bronchoscopic photograph of same patient with Irregular growth in left main bronchus which on bronchial biopsy is suggestive of squamous cell carcinoma.

Figure 3 is showing chest rontgenograph having perihilar consolidation in left lower zone and Figure 4 is bronchographic photograph of same patient with large blood clot resulting in $90 \%$ occlusion of apical segmental bronchus of left lower lobe.

CT-guided FNAC is a good procedure for peripherally situated lesions when FOB result is inconclusive. Its diagnostic yield is significantly high, and in our series CT guided bopsy was helpful in diagnosis of $91.6 \%$ patient. Serological tests or biopsies of extrapulmonary sites are helpful for diagnosis in some case. Last resort for confirmatory diagnosis is surgical (open or VATS) biopsy to diagnose refractory or nonresolving "pneumonias. In our series two undiagnosed patients did not gave consent for open biopsy. 


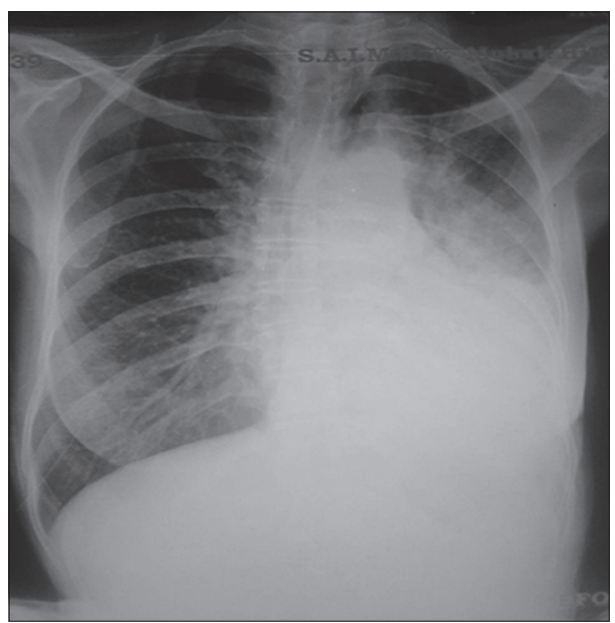

Figure 1: Consolidation left lower Lobe

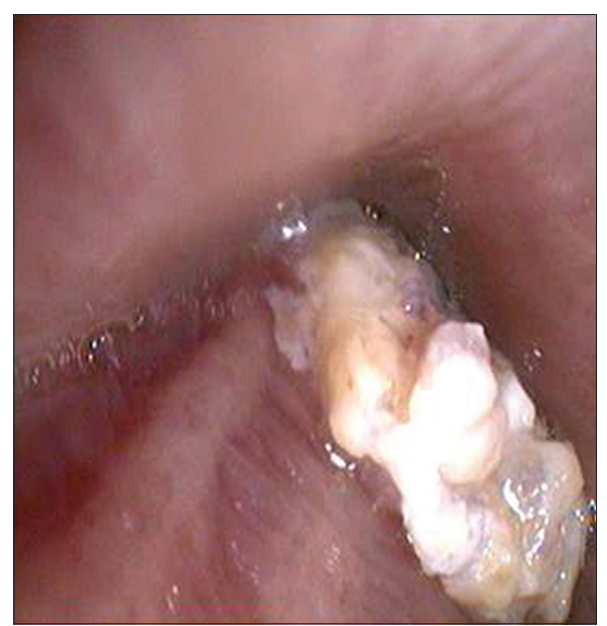

Figure 2: Irregular growth in Left main bronchus squamous cell Malignancy

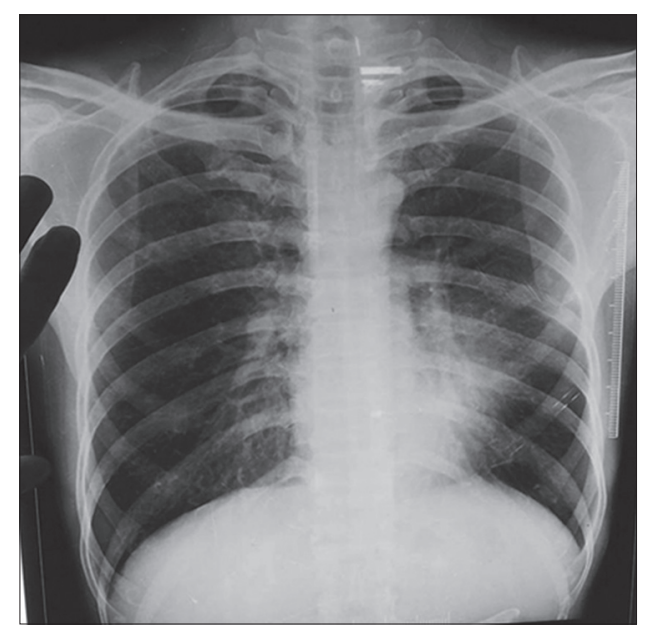

Figure 3: Left Perihilar consolidation

The shortcoming of our study was that CT guided Biopsy was done only in twelve selected cases where lesion was peripheral and FOB result were inconclusive.

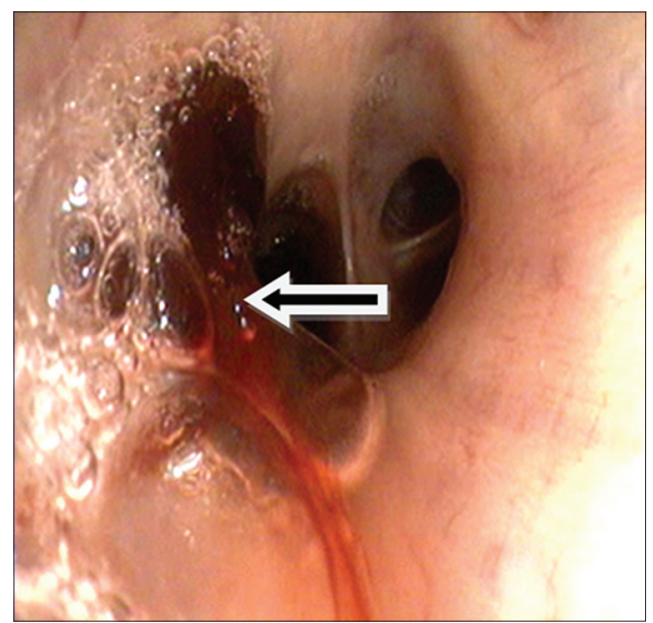

Figure 4: Blood Clot in left apical segmental bronchus of left lower lobe

The diagnostic efficacy of FOB was $81 \%$ and diagnostic efficacy of CT guided Biopsy was 91\%. Both procedure are safe and no mortality was reported.

One patient had mild hemoptysis after CT guided FNAC which was managed conservatively and one patient had pneumothorax following TBLB which was managed successfully with intercostal tube drainage and intercostal tube was removed after 3 days when pneumothorax was resolved.

To summarize, treating non-resolving pneumonia is difficult to treat situation. Comorbidities like COPD, diabetes, alcoholism, smoking, Bronchiectasis and immunosuppression are significant factors causing nonresolution. Pyogenic infections are the commonest cause of Non resolving pneumonia. Tuberculosis should be strongly suspected in patient of uncontrolled diabetes mellitus with non resolving pneumonia. For centrally arising lung malignancy FOB with endobronchial biopsy, TBLB and BAL cytology is very useful. For peripherally arising lung malignancy CT guided FNAC is useful.

Now invasive procedures are performed more frequently to reach a final diagnosis. Both FOB and $\mathrm{CT}$ guided FNAC is a very useful investigation to diagnose cause of non resolving pneumonia. CT-guided FNAC gives good yield specially in peripherally situated non resolving pneumonia.

\section{ACKNOWLEDGEMENT}

We are very thankful to our chairmen SAMC \& PG institute Indore M.P. (India) Dr. Vinod Bhandari for wonderful guidance and all the contribution to our study. 


\section{REFERENCES}

1. Kirtland $\mathrm{SH}$ and Winterbauer $\mathrm{RH}$. Slowly resolving chronic and recurrent Pneumonia. Clin Chest Med 199l; 12:303-318.

2. Fein $\mathrm{AM}$, Feinsilver $\mathrm{SH}$ and Niederman MS. Non-resolving and slowly resolving pneumonia. Diagnosis and management in the elderly patient. Clin Chest Med 1993; 14:555-569.

3. Fein $\mathrm{AM}$ and Feinsilver $\mathrm{SH}$. The approach to non-resolving pneumonia in the elderly. Semin Respir Infect 1993; 8:59-72.

4. Fein AM. Pneumonia in the elderly: overview of diagnostic and therapeutic approaches. Clin Infect Dis 1999; 28:726-729.

5. Marrie TJ. Mycoplasma pneumoniae pneumonia requiring hospitalization, with emphasis on infection in the elderly. Arch Intern Med 1993; 153:488-494.

6. Weyers $\mathrm{CM}$ and Leeper KV. Nonresolving pneumonia. Clin Chest Med 2005; 26:143-158.

7. Gleichman TK, Leder MM, Zahn DW. Major etiologic factors producing delayed resolution in pneumonia. Am J Med Sci 1949; 218:309-320.

8. Israel HL, Weiss W, Eisenberg GM, Strandness DE and Flippin
HF. Delayed resolution of pneumonia. Med Clin North Am 1956; 40:1291-1303.

9. Mafarlane JT, Miller AC, Roderick Smith WH, Morris AH and Rose $\mathrm{DH}$. Comparative radiographic features of community acquired legionnaires' disease, pneumococcal pneumonia, mycoplasma pneumonia, and psittacosis. Thorax 1984; 39:2833.

10. Stephen JJ, Waldemar GJ and Alan KP. The radiographic resolution of Streptococcus pneumonia. N Engl J Med 1975; 293:798-801.

11. Souweine B, Verber B, Bedos JP, Gachot B, Dombret MC, Regnier B and Wolff M. Diagnostic accuracy of protected specimen brush and bronchoalveolar lavage in nosocomial pneumonia: impact of previous antimicrobial treatments. Crit Care Med 1998; 26:236-244.

12. Rodrigues J, Niederman MS, Fein AM and Pai PB. Nonresolving pneumonia in steroid-treated patients with obstructive lung diseases. Am J Med 1992; 93:29-34.

13. Feinsilver SH, Fein AM, Niederman MS, Schult DE and Faegenburg $\mathrm{DH}$. Utility of firberoptic bronchoscopy in non resolving pneumonia. Chest 1990; 98:1322-1326.

\section{Authors Contribution:}

BKJ - Designed the study, performed the laboratory tests, analysed the data, drafted the manuscript and reviewed the manuscript; PS - Contributed to the study design; AB - Reviewed the manuscript; SM - Reviewed the manuscript; NP - Designed the study; DP - Designed the study.

Source of Support: Nil, Conflict of Interest: None declared. 